\title{
Career lifetime advances in nuclear medicine
}

\author{
Andrew Hilson
}

When I qualified in 1967, medicine was much simpler, but becoming more interesting. There were new diuretics (ethacrynic acid and furosemide) which really worked - I'm probably one of the last housemen to have used Southey's tubes for drainage of intractable oedema. We also had new antibiotics such as ampicillin. My surgical skills were minimal, and my first house job was as house surgeon to the cardiothoracic unit at Westminster Hospital, where I clerked the patients, held the retractor and looked after the patients in the postoperative period. This was basically applied physiology - and I enjoyed it. My medical house job was in cardiothoracic medicine, and here I came across my first real taste of what was to become nuclear medicine when we were able to send patients over to University College Hospital (UCH) to have their pulmonary perfusion assessed. ${ }^{1}$ Remember, there was no computed tomography (CT), no magnetic resonance imaging (MRI), no ultrasound and little angiography.

I enjoyed applied physiology, so I managed to persuade the cardiothoracic unit that they needed a senior house officer in what would now be called intensive care, and they persuaded the Garfield Weston Foundation to fund a post. This included looking after postoperative patients, as well acting as resident doctor to the respiratory and coronary care units (including two hyperbaric tanks). I also found time to go on the course in clinical measurement run by Dr Percy Cliffe's department, and to teach myself programming in FORTRAN on the IBM 1800 computer.

Having decided that a physician's life was for me, I went to work for Dr Phillip Harvey at St Stephen's Hospital in Fulham, where I passed MRCP part I, and took part II. I then decided that to be a general physician I needed to learn more neurology, so I got a registrar post in neurology at the Royal Free Hospital - where nuclear medicine re-entered my life. The standard methods of diagnosing intracerebral pathology involved basic ultrasound to look for a shift of the midline structures, followed by angiography, usually by direct carotid puncture. If this did not resolve the issue, the patient went to Queens Square for a pneumoencephalogram. However, the medical physics department at the Royal Free had a rectilinear scanner, and one of my tasks was to inject the patients with the pertechnetate to allow imaging. The images were amazing - it was possible to see a tumour without risk to the patient. I also helped set up and run a Parkinson's disease clinic for trials of L-DOPA and related agents. ${ }^{2}$

Still trying to be a general physician, I went on to a short spell as a locum senior registrar in rheumatology, and then was offered a locum post at Guy's in endocrinology and nuclear medicine. These were two specialties I had not tried, so I

Andrew Hilson, Harveian librarian, Royal College of Physicians, London accepted the offer. The nuclear medicine was a revelation. There was exposure to a wide new field. We were measuring glomerular filtration rate using Cr-51-EDTA, looking for pulmonary emboli, carrying out renography using probes to assess kidney function, and localising the placenta in pregnant patients with antepartum haemorrhage using a Sn/In-1113m generator, a probe and a calibrated tea towel. I knew that this felt right for me and there was a new post of senior registrar in nuclear medicine about to be advertised. I was a bit hesitant at this career change from general medicine and, when the locum came to an end, I went to do a locum in general practice for three months. However, this confirmed that nuclear medicine was for me and I applied for, and got, the post.

This was a fascinating opportunity. Nuclear medicine as a specialty was exploding, and the unit was in the fortunate position of being in a new building with a guaranteed income from an anonymous donation. Initially the star piece of equipment was a rectilinear scanner which could get a whole-body image onto a single sheet of film. The images were a bit grainy, but could be improved by using a 'minifying' glass. The major use of this was for bone imaging, using F-18- fluoride which came from the Medical Research Council cyclotron unit at the Hammersmith Hospital. F-18 only has a half-life of 90 minutes, and we would have four patients lined up ready to be injected, but if the traffic was bad, one would be sent away. We also ran the thyroid clinic, where we diagnosed and treated patients with thyrotoxicosis and thyroid cancer.

Shortly after we moved into the new unit, we took delivery of a wonderful new gamma camera. The principle of the gamma camera had been described by Hal Anger, but existing cameras only had a maximum of 19 photo-multiplier tubes, had very poor resolution, and were impossible to keep in tune. The OhioNuclear 100 had 37 tubes across its 10-inch crystal, with an alternative circuitry which made for better resolution and uniformity. Images were recorded onto Polaroid film. Because the data were recorded and displayed without the camera moving, it was possible to acquire serial dynamic images by pulling the films from the cassette by hand. This required a very good technician with a firm grip wearing surgical gloves, and another technician unpeeling the films after the correct development time and varnishing them.

Using this camera, we were able to get dramatically improved images. Thyroid nodules could be clearly resolved. We used labelled colloid to image the liver, and were often able to separate diffuse liver disease from metastatic disease. There was a steady demand for my old friend brain imaging and, using a combination of dynamic and delayed imaging, we could usually diagnose cerebrovascular attacks, tumours and subdural haematomas. The better resolution and lower dose meant that 
imaging of the placenta with labelled autologous red cells was more reliable.

The combination of the development of Tc-99m-phosphonates and better resolution of the gamma camera produced an explosion in bone imaging. A discussion with a an orthopaedic trainee led to the realisation that uptake of the tracer into fractures might be of clinical value. ${ }^{3}$

Later we added a O-N 110 camera with a larger crystal, which allowed us to image both lungs at the same time, and V/Q imaging was further improved by the regular delivery of a $\mathrm{Kr}$ $81 \mathrm{~m}$ generator from the Hammersmith Hospital.

We also acquired a dedicated computer system, based on a PDP11 computer with a whole $16 \mathrm{k}$ of memory and two disk drives, each holding a massive $256 \mathrm{k}$. The images were displayed on a persistence oscilloscope, and photographed onto Polaroid film using a hand-held camera with a long snout.

Guy's had a major renal unit, with a strong interest in renal transplantation, and I became involved in this area. Renal biopsy was difficult and rejection was common. I worked on the problem of detecting the changes in transplant blood flow associated with rejection, using this computer system and the gamma camera. We were able to show that this was clinically useful, ${ }^{4}$ and very soon we were doing 10 or 12 studies three days a week.

One area we couldn't get to work was pancreatic imaging. The technique was based on using Se-75-selenomethionine, a labelled amino acid, which is taken up by both the liver and pancreas. By subtracting a liver image obtained using radioactive gold colloid, an image of the pancreas was obtained. The experts in this were the Royal Free, and we used to send patients there for further treatment.

One test which we could get to work, but didn't promote, was for the detection and quantitation of gastrointestinal blood loss. This involved labelling the patient's red blood cells with Cr-51, reinjecting them, and measuring the radioactivity which appeared in a seven-day stool collection. I won't go into the details, but it involved paint tins, a shaker and added a whole new meaning to 'radioactive decay'.

Ultrasound soon emerged so we were able to give up placental localisation. More seriously, it was better than we were at detecting liver metastases, so was the specialty finished? No. CT was developed, which was better than we were at studying the brain, so was the specialty finished? No. We moved into cardiology. We started with the observation that bone imaging agents were taken up into recent infarcts. This was then followed by the use of $\mathrm{Tl}-201$, a potassium analogue for imaging of myocardial blood flow. The images were a major advance in cardiology, and our workload expanded to fill the gap. This was accompanied by the development of radionuclide ventriculography. The cardiologists said that all they needed to assess the heart was the ejection fraction. We gated cardiac blood pool images from the R-wave of the electrocardiogram to get end-systolic and end-diastolic images and the left ventricular ejection fraction (LVEF). The cardiologists said that all they needed to assess the heart was $\mathrm{dV} / \mathrm{dt}$ of the left ventricle. Along came the concept of splitting the cardiac cycle into 16 gated segments using a computer. This gave us a more accurate LVEF, as well as $\mathrm{dV} / \mathrm{dt}$.

My career moved on. I became a consultant working part time at the Royal Free Hospital and part time at the Institute of Urology in Covent Garden. This coincided with a major development - 3D imaging. A Scandinavian group used a gamma camera which had a rotating mount to show that by rotating it around the patient it was possible to obtain a series of projection images which could be reconstructed in a manner analogous to CT. It was slow - when we started it took 45 minutes to reconstruct a cardiac image, but single photon emission computed tomography (SPECT) was clearly an advance. Using Tl-201 it was difficult, as the agent redistributed through the potassium space, but Tc-99m-labelled cardiac agents were developed which solved that problem. SPECT was also a major advance in bone imaging.

Other areas were also moving ahead. I-131-MIBG was developed for studying the adrenal medulla. It was soon realised that it could be used for localising neuroblastoma. Then it was realised that higher activities could be used for therapy. This was the start of a (slow) explosion in therapeutic nuclear medicine. We began to realise that neuroendocrine tumours were more common than we thought, and some could be treated with I-131-MIBG.

The workload continued to increase, and I moved full time to the Royal Free. The combination of the liver work and the skills available in interventional radiology led us to the use of I-131Lipiodol for hepatocellular carcinoma. ${ }^{5}$ In turn, our therapy practice led us to be become further involved in the diagnosis and treatment of neuroendocrine tumours, which are best treated by multidisciplinary teams. ${ }^{6,7}$

MRI came along, which was clearly going to end nuclear medicine. Now the technique of positron emission tomography (PET), which I had learnt about in the 1970s on my MSc course, reappeared. However, the technology had advanced, and the concept of adding a CT scanner to the machine for attenuation correction and localisation (PET/CT) revolutionised the field. This was followed later by the application of the same approach to SPECT to give us the SPECT/CT system.

We also worked on the use of Tc-HIDA agents for imaging the biliary tract $^{8}$; penile blood flow - the 'isotopic phallogram ${ }^{9}$ (rendered irrelevant by Viagra); ureteric peristalsis ${ }^{10,11}$; and oesophageal motility in scleroderma. ${ }^{12}$

So, what are the latest techniques I have had to learn? Imaging of dopamine transporters for the diagnosis and staging of Parkinson's disease, and the use of F-18-fluoride for bone lesions - both issues from my early career.

How to summarise nuclear medicine? It is applied physiology, studied in human beings. It needs a good clinical training. It's been fun. What more could I have asked for?

\section{References}

1 Secker-Walker RH. Scintillation scanning of lungs in diagnosis of pulmonary embolism. BMJ 1968;27:206-8. 
2 Calne DB, Reid JL, Vakil SD et al. Idiopathic Parkinsonism treated with an extracerebral decarboxylase inhibitor in combination with levodopa. BMJ 1971;3:729-32.

3 Hilson AJW, Elsayed TF, Saunders AJS, Maisey MN. Use of radioisotope imaging in the diagnosis of stress and scaphoid fractures. Br J Radiol 1979;52:248-9.

4 Hilson AJW, Maisey MN, Brown CB, Ogg CS, Bewick MS. Dynamic renal transplant imaging with Tc-99m DTPA (Sn) supplemented by a transplant perfusion index in the management of renal transplants. $J$ Nucl Med 1978;19:994-1000.

5 Bhattacharya S, Novell JR, Dusheiko GM et al. Epirubicin-Lipiodol chemotherapy versus 131 iodine-Lipiodol radiotherapy in the treatment of unresectable hepatocellular carcinoma. Cancer 1995;76:2202-10.

6 Caplin ME, Buscombe JR, Hilson AJ et al. Carcinoid tumour. Lancet 1998;352:799-805.

7 El Haddad KS, Dick ER, Tibballs JM et al. How should we assess the liver in patients with neuro-endocrine tumours? A comparison of triple-phase helical CT, I-123-MIBG SPECT and In-111-octreotide SPECT. Radiology 2000;217:367.
8 Hilson AJW, Evans G, Hobbs KEF. Tc-99m-Bromotrimethyl HIDA (Mebrofenin) a clinical assessment. Euro J Nucl Med 1985;11:A37.

9 Siraj QH, Hilson AJW, Townell NH, Morgan RJ, Cottrall MF. The role of the radioisotope phallogram in the investigation of vasculogenic impotence. Nucl Med Commun 1986;7:173-82.

10 Woolfson RG, Lewis CA, Hill PD, Hilson AJW, Neild GH. Ureteric peristalsis studies in loin pain and haematuria syndrome: another diagnostic disappointment. Br J Urol 1993;72:291-2.

11 Lewis CA, Coptcoat MJ, Carter SS et al. Radionuclide imaging of ureteric peristalsis. Br J Urol 1989;63:144-8.

12 Kaye SA, Siraj QH, Agnew JE, Hilson AJW, Black CM. Detection of early asymptomatic esophageal dysfunction in systemic sclerosis using a new scintigraphic grading method. J Rheumatol 1996;23:297-301.

Address for correspondence: Dr A Hilson,

Royal College of Physicians, 11 St Andrews Place,

Regent's Park, London NW1 4LE.

Email: andrew.hilson@rcplondon.ac.uk

\section{Working party report}

\section{Local adult neurology services for the next decade Report of a working party}

\begin{abstract}
Neurological disorders are very common, accounting for about one in ten general practitioner consultations, around $10 \%$ of emergency medical admissions (excluding stroke) and disability for one in $\mathbf{5 0}$ of the UK population.
\end{abstract}

Patients require access to different parts of the neurological care pathway at different stages of their illness (acute admission, outpatient care and longterm care). However, these are currently poorly planned and organised. Good management requires better integrated primary, secondary and tertiary resources to achieve a neurology network that is easily accessible, provides local care where appropriate and, when necessary, involves the regional neurosciences centre.

Highlighting the need for acute neurology services in district general hospitals and the community, this report emphasises the value of multidisciplinary interaction with nurses and GPs, and urges a better structured and integrated approach. Other improvements proposed include a rise in the number of district general hospital neurologists, and dedicated nursing staff and neurology wards. The report also stresses the need for neurologists to take an active role in the planning and commissioning of services.

Published: May 2011 ISBN: 9781860164446

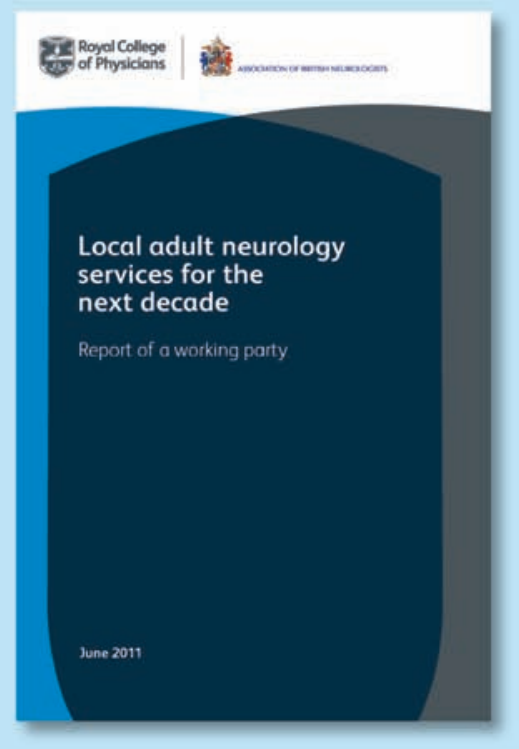

Price: $£ 5.00$ UK, $£ 7.00$ overseas (inc post and packing) 